\title{
A Produção Científica nos Anais do Encontro Nacional de Engenharia de Produção: Um Levantamento de Métodos e Tipos de Pesquisa \\ PRODUÇÃO
}

\author{
Rosa Maria Villares. S. Berto - rosamvsb@usp.br \\ Davi Noboru Nakano-dnnakano@usp.br \\ Escola Politécnica da Universidade de São Paulo - Departamento de Engenharia de Produçāo \\ Av. Prof. Almeida Prado, 128, trav.2 - CEP 05508-900 - São Paulo - SP \\ Au Prof. Almeida Prado, 128, trau.2 - CEP 05508-900 - Säo Paulo- SP
}

Resumo

O trabalho analisa os métodos e técnicas de pesquisa descritos nos relatos publicados nos Anais do ENEGEP - de 1996 a 1998. Utilizando a tipologia proposta por Filippini (1997), os trabalhos foram classificados de acordo com o tipo de pesquisa utilizado e os resultados foram comparados aos encontrados por Filippini nos anais do congresso do DSI. As principais conclusōes sāo: incentivo a um maior número de trabalhos com pesquisa de campo e a necessidade de aprofundamento e maior rigor metodológico no planejamento e na conduçāo das pesquisas.

\section{Abstract}

This paper analyses the research approaches and methods used in the papers of the proceedings of the 1996, 1997 and 1998 ENEGEP's. Starting from a typology developed by Filippini (1997), the research methods used were classified and compared with the results found by Filippini in the proceedings of the DSI conference. The main conclusions point toward the need of deeper methodological concern and better use of research methodos and to more empirical-based research.

Palavras Chaves:

Engenharia de Produçāo, Metodologia Científica, Métodos de Pesquisa.

Keywords:

Operations management, research methods, cientific methodology.

\section{Introdução}

Este trabalho pretende dar continuidade às discussões sobre o uso da Metodologia Científica nos trabalhos de pesquisa em Engenharia de Produção, que vêm sendo desenvolvidas no Departamento de Engenharia de Produção da EPUSP. A partir de reflexões teórico-conceituais sobre o tema (Nakano e Fleury, 1996), foi realizado um levantamento acerca dos métodos de pesquisa utilizados nas teses e dissertações defendidas no referido Departamento (Berto e Nakano, 1998) e que, neste trabalho, tem seu campo de análise expandido, na busca de uma visão mais abrangente sobre assunto, desta feita, em âmbito nacional.

Tendo como pressuposto que os Encontros Nacionais de Engenharia de Produção, por seu alcance, abrangência e relevância, refletem o estado da arte da pesquisa brasileira na área, foi efetuado um levantamento dos tipos de pesquisa utilizados em trabalhos publicados nos anais dos três últimos ENEGEP's (1996 a 1998), com o objetivo de constatar tendências e/ou corroborar resultados como os obtidos nos estudos anteriores.

Para a realização do levantamento foi utilizada uma classificação desenvolvida e aplicada por Filippini (1997), em pesquisa similar internacio- 
PRODUÇÃO

nal, possibilitando a comparação entre os resultados encontrados nos dois estudos.

\section{Vigência dos métodos e tipos de pesquisa}

As abordagens de pesquisa são condutas que orientam o processo de investigação, são formas ou maneiras de aproximação e focalização do problema ou fenômeno que se pretende estudar, prestando-se à identificação dos métodos e tipos de pesquisa adequados às soluções desejadas. Dependem da natureza do problema e de sua formulação, da teoria de base e referencial teórico-cultural que o sustentam e da proximidade do pesquisador com o.objeto de análise. Criatividade na percepção da relevância dos dados e das relações entre variáveis é requerida e desejável. Condicionam a forma como os projetos ou planos de investigação são formulados: sistematizam e orientam a revisão bibliográfica, a coleta e a análise de dados, assim como a discussão dos resultados (Lakatos e Marconi, 1991, Cervo e Bervian, 1996).

As abordagens de pesquisa tradicionais são as chamadas quantitativas, com natureza empírica e hipóteses "fortes" e bem formuladas. Baseiamse em métodos lógico-dedutivos, buscam explicar relações de causa/efeito e, através da generalização de resultados, possibilitar replicações. Privilegiam estudos do tipo "antes e depois", propiciando análises estáticas e instantâneas da realidade, como se fossem fotografias. Têm ainda como características de conduta de investigação, a seleção das amostras, a distância ou a ausência de contato entre o pesquisador e o objeto de estudo. Os relatos dos resultados obtidos devem ser objetivos e primar por descrições externas e metrificadas. Os tipos de pesquisa inerentes às abordagens quantitativas são os levantamentos (surveys), os estudos teórico-conceituais, os diagnósticos, as modela- gens e simulações, que recriam artificialmente a realidade mediante dados quantitativos.

As pesquisas de natureza qualitativa buscam aproximar a teoria e os fatos, através da descrição e interpretação de episódios isolados ou únicos, privilegiando o conhecimento das relação entre contexto e ação (método indutivo). Através de análises fenomenológicas e da subjetividade do pesquisador chegam, geralmente, a resultados particularizados que possibilitam, no máximo a comparação entre casos.

Nessa ótica, a delimitação do problema de pesquisa inclui uma visão de contexto histórico ou de desenvolvimento, enfatizando o processo dos acontecimentos e a seqüência dos fatos ao longo do tempo. O enfoque da pesquisa pode ser mais desestruturado, não havendo necessidade, conforme o método empregado, de hipóteses fortes no início da pesquisa, conferindo-lhe bastante flexibilidade. A objetividade dá lugar ao subjetivismo do pesquisador, que retrata a realidade do fenômeno através da sua dinâmica interna, como se fora um filme ou um objeto tridimensional. A proximidade e o contato do pesquisador com o objeto de análise propiciam a elaboração de relatos e depoimentos que privilegiam aspectos internos e particulares da situação. Nas abordagens qualitativas os tipos de pesquisa mais freqüentes são: estudo de caso, observação participante, pesquisa participante e pesquisa ação.

As hipóteses são os fatores produtivos da pesquisa - principalmente as de abordagem quantitativa - e desencadeiam o processo científico. São como soluções provisórias ao problema, servem para orientar a investigação e necessitam teste, verificação, focalizando os segmentos a serem observados, evitando dispersões. Além disso, identificam as informações necessárias à explicação dos fenômenos ao desencadearem inferências sobre os dados - seu caráter, quanti- 
dade e relações - classificando-os e estabelecendo relações de importância entre as variáveis envolvidas no processo. Trata-se de um importante instrumento de trabalho da teoria ao relacioná-la a fatos e dados. Desta maneira, as hipóteses influenciam as abordagens e determinam outros meios operacionais de pesquisa. Podem ainda explicar ou "proteger" outras hipóteses. Nas abordagens qualitativas as hipóteses são em geral "fracas" ao início das pesquisas (principalmente nas de origem sociológica ou antropológica), por não estarem amparadas definitivamente por leis e teorias.

As variáveis são predicados, atributos, propriedades, características e/ou valores mensuráveis encontrados nos objetos de estudo. Servem para testar a(s) relação(ões) de massa, velocidade, energia, idade, nível social, financeiro e educacional, valor monetário, classe social, sexo, índices de qualidade, produtividade, entre outras, que sejam necessárias para a verificação das hipóteses formuladas.
Como se vê, as investigações científicas têm caráter e natureza variados, quando se trata de estabelecer, contextualizar e relacionar aspectos diferentes de um ou mais fenômenos ou situações-problema, organizando informações e consolidando o conhecimento. Podem assumir caracteres variados, em função da intenção que se apresenta: prospectiva, preditiva, retrospectiva, descritiva, explanatória, etiológica, exploratória ou de investigação.

\section{Tipos de pesquisa nos anais do ENEGEP}

A utilização das diferentes abordagens e tipos de pesquisa em Engenharia de Produção é um tema que tem suscitado discussões em âmbito internacional. Por exemplo, um levantamento realizado sobre artigos publicados em Gestão de Operações nos Estados Unidos e no Reino Unido, no período de 1985 a 1994, (Voss, 1995 apud Filippini e Voss, 1997), cujos resultados são mostrados na Tabela 1, mostrou em primeiro

\begin{tabular}{ccc}
\hline Posição & Estados Unidos & Reino Unido \\
\hline $\mathbf{1}$ & Modelagem & Conceitual \\
$\mathbf{2}$ & Simulação & Estudos de Campo \\
$\mathbf{3}$ & Conceitual & Survey \\
$\mathbf{4}$ & Survey & Modelagem \\
$\mathbf{5}$ & Estudos de Campo & Simulação \\
\hline
\end{tabular}

Tabela 1: Freqüência de trabalhos de pesquisa por tipo de pesquisa

Fonte: Filippini e Voss, 1997 
PRODUÇÃO

lugar, um grande contraste entre os tipos de pesquisa utilizados nos dois países e a ausência de base empírica nos trabalhos publicados em revistas norte-americanas. A partir desse tipo de constatação, o interesse em avaliar a pesquisa nacional foi imediato. Para isso, planejou-se o levantamento dos tipos de pesquisa utilizados nos trabalhos apresentados no ENEGEP.

Para a realização do levantamento dos tipos de pesquisa utilizados nos trabalhos publicados nos Anais dos ENEGEP's, no período de 1996 a 1998, foi utilizada a tipologia proposta por Filippini (1997). Para o seu desenvolvimento, o autor trabalha duas dimensões de análise e comparação: a dimensão da geração de conhecimento, cujos extremos são o método dedutivo e o indutivo, com o empiricismo ocupando uma posição intermediária entre os dois extremos. A outra dimensão refere-se à fonte e tipo de informação utilizadas, que variam da observação direta e mensurável de um fato até o subjetivismo do pesquisador (reconstrução artificial da realidade), onde a observação fundada na percepção pessoal de um indivíduo, ocupa uma posição intermediária entre esses dois extremos.
A partir dessas duas dimensões, pode-se classificar os tipos de pesquisa. Assim a modelagem, isto é, o uso de estratégias artificiais para reconstruir a realidade através do uso de lógica dedutiva, se coloca em um dos quadrantes superior direito - definidos pelas referidas dimensões (Figura 1). Estudos conceituais, como o desenvolvimento de tipologias e taxonomias, podem ser classificados como métodos artificiais com o uso de lógica indutiva. Os estudos de caso ocupam o quadrante destinado à observação direta da realidade com o uso de lógica indutiva. Outros tipos de pesquisa ocupam posições intermediárias no plano dimensional como os resultantes da percepção pessoal e observação direta do pesquisador sobre a realidade objetiva ou aqueles baseados no empiricismo, como o levantamento (survey) e o diagnóstico.

A seguir, a Tabela 2 detalha a ocorrência de cada um dos tipos de pesquisa nos anais da conferência do Decision Science Institute, dos anos de 1986/87 e 1996 (Filippini, 1997). Filippini observa que houve queda na quantidade de estudos baseados em Modelagem e Simulação e um crescimento concomitante dos estudos TeóricoConceituais e os estudos de base empirica, especialmente os surveys.

\begin{tabular}{|c|c|c|}
\hline & Lógica dedutiva & Lógica Indutiva \\
\hline $\begin{array}{c}\text { Reconstrução artificial } \\
\text { da realidade }\end{array}$ & Modelagem & $\begin{array}{c}\text { Modelagens Conceituais } \\
\text { (Taxonomias) }\end{array}$ \\
\hline $\begin{array}{c}\text { Observação direta da } \\
\text { realidade }\end{array}$ & & Estudos de Caso \\
\hline
\end{tabular}

Figural: Dimensões dos tipos de pesquisa

Fonte: adaptado de Filippini, 1997 


\begin{tabular}{lcc}
\hline Tipo de Pesquisa & Anais 1986/87 & Anais $\mathbf{1 9 9 6}$ \\
\hline Modelagem & $33,96 \%$ & $27,66 \%$ \\
Simulação & $32,08 \%$ & $16,17 \%$ \\
Survey & $13,21 \%$ & $26,81 \%$ \\
Estudo de Caso & $7,54 \%$ & $2,13 \%$ \\
Estudo de Campo & $1,89 \%$ & $5,53 \%$ \\
Experimento & $1,89 \%$ & $0,85 \%$ \\
Teórico / Conceitual & $9,43 \%$ & $20,85 \%$ \\
\hline
\end{tabular}

Tabela 2: Tipos de Pesquisa utilizados nos Anais do DSI, 1986/87 e 1996.

Fonte: Filippini (1997)

O objetivo deste artigo é efetuar um levantamento semelhante ao realizado por Filippini, desta feita nos anais do ENEGEP dos três últimos anos. Apesar da discussão conceitual que realizou, descrevendo as dimensões utilizadas para a definição dos tipos de pesquisa, Filippini não detalhou as características consideradas, por exemplo, na definição de Estudo de Campo. Assim, para que um levantamento semelhante fosse efetuado, de forma que os resultados fossem comparáveis aos apresentados por ele, foram feitas as seguintes considerações acerca dos tipos analisados:

- Modelagem: Uso de técnicas matemáticas para descrever o funcionamento de um sistema ou de parte de um sistema produtivo.

- Simulação: Uso de técnicas computacionais para simular o funcionamento de sistemas produtivos, a partir de modelos matemáticos.

-Survey: Uso de um único instrumento de coleta de dados (em geral um questionário), aplicado a amostras de tamanho grande, com o uso de técnicas de amostragem e análise estatística.
- Estudo de Caso: Análise aprofundada de um ou mais objetos (casos), com o uso de múltiplos instrumentos de coleta de dados e interação entre o pesquisador e o objeto de pesquisa.

- Estudo de Campo: Outros métodos de pesquisa (principalmente de enfoque qualitativo). Presença de dados de campo, sem estruturação formal do método de pesquisa.

- Experimento: Estudo da relação causal entre duas variáveis de um sistema sob condições controladas pelo pesquisador.

- Teórico / conceitual: Discussões conceituais a partir da literatura, revisões bibliográficas. Modelagens conceituais, baseadas na percepção e experiências do autor.

Foram analisados todos os trabalhos que puderam ser observados/consultados nos anais dos três eventos realizados. É importante notar que foram encontradas algumas situações atípicas como: trabalhos repetidos (nos anais de 1998) e trabalhos que não analisados devido a problemas apresentados pela mídia utilizada no seu registro (CD-ROM, principalmente de 1996). Por esse motivo o total de artigos analisados não corresponde ao total declarado de trabalhos nos Anais dos congressos. 
PRODUÇ̃̃O

No levantamento realizado, não houve julgamento de mérito quanto à escolha do tipo de pesquisa. Desta forma, quando houve declaração explícita do autor nesse aspecto, ela foi aceita sem contestação, mesmo quando havia clara $\mathrm{e}$ evidente incompatibilidade entre a tipologia declarada e a efetivamente praticada. Tampouco foi analisada a adequação entre a finalidade e tipo de pesquisa ao problema ou tema propostos.

Na categoria Estudos de Campo foram classificados os trabalhos que fizeram uso de, por exemplo: entrevistas (sem o aprofundamento necessário de um estudo de caso), uso concomitante de mais de um tipo de pesquisa (triangulação), pesquisa participante, pesquisa ação, tipos específicos de análise (análise ergonômica do trabalho), coleta de dados de campo sem estrutura ou declaração sobre o tipo de pesquisa ou método empregado. Os resultados obtidos foram os seguintes:

\begin{tabular}{c|cc|cc|cc|cc}
\hline & 1996 & $\%$ & 1997 & $\%$ & 1998 & $\%$ & Total & $\%$ \\
\hline Modelagem & 76 & 12,69 & 78 & $16,49 \%$ & 67 & $12,45 \%$ & 221 & $13,73 \%$ \\
Simulação & 16 & $2,67 \%$ & 20 & $4,23 \%$ & 18 & $3,35 \%$ & 54 & $3,35 \%$ \\
Survey & 18 & $3,01 \%$ & 23 & $4,86 \%$ & 26 & $4,83 \%$ & 67 & $4,16 \%$ \\
Estudo de Caso & 101 & $16,86 \%$ & 67 & $14,16 \%$ & 75 & $13,94 \%$ & 243 & $15,09 \%$ \\
Estudo de Campo & 89 & $14,86 \%$ & 92 & $19,45 \%$ & 89 & $16,54 \%$ & 270 & $16,77 \%$ \\
Experimento & 9 & $1,50 \%$ & 11 & $2,33 \%$ & 4 & $0,74 \%$ & 24 & $1,49 \%$ \\
Teórico Conceitual & 290 & $48,41 \%$ & 182 & $38,48 \%$ & 259 & $48,14 \%$ & 731 & $45,40 \%$ \\
& 599 & & 473 & & 538 & & 1610 & \\
\hline
\end{tabular}

Tabela 3: Tipos de Pesquisa utilizados nos trabalhos dos Anais ENEGEP 96-98.

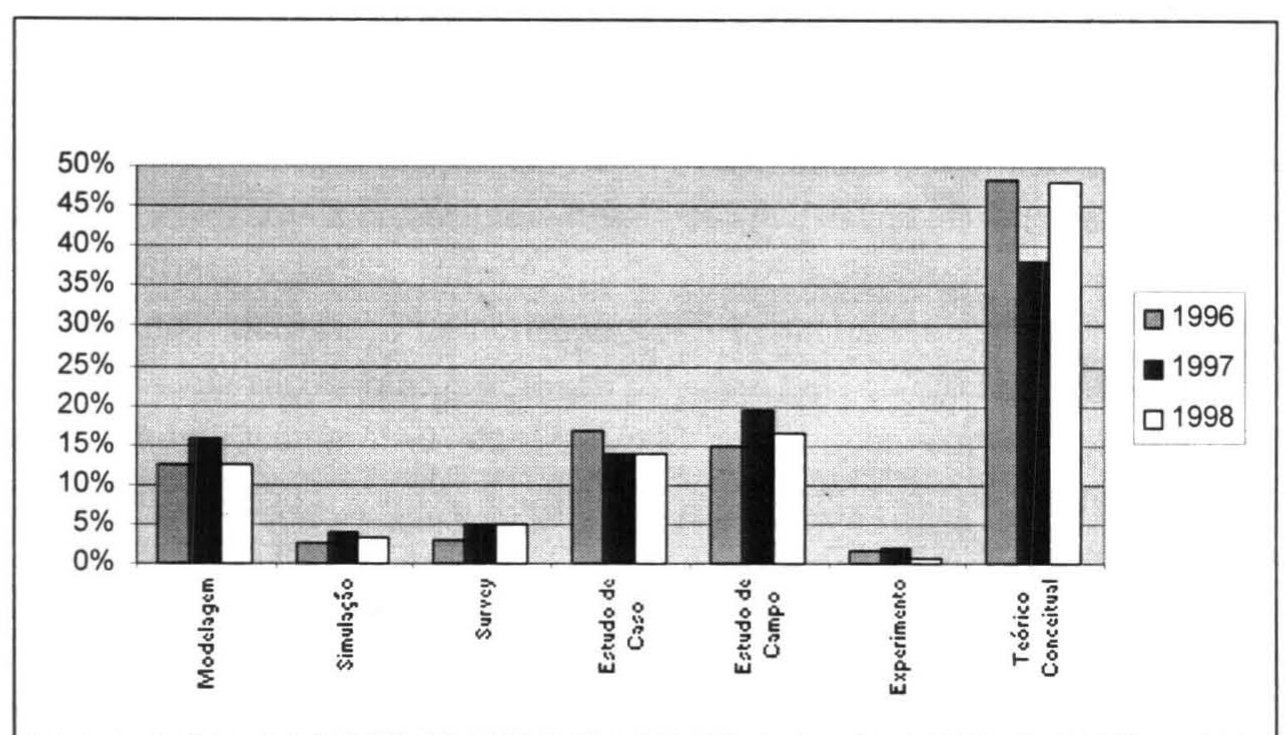

Figura 2: Ocorrência dos tipos de pesquisa utilizados nos trabalhos publicados nos Anais

96-98, em valores percentuais 
4. Discussão dos resultados

A ocorrência de estudos teórico-conceituais foi marcante e destacada se comparada aos demais tipos de pesquisa. Nessa categoria foram classificados os trabalhos de revisão bibliográfica, as discussões conceituais sem dados de campo e as análises de dados secundários, ou seja, obtidos de fontes que não as diretas, bem como os de modelagem conceitual (por exemplo, discussões sobre o uso do TQM, QFD, JIT, etc.). O volume exacerbado de estudos teórico-conceituais pode revelar que a maioria dos trabalhos publicados reflete estágios iniciais de pesquisas, onde os autores estão ainda delimitando o objeto, as variáveis e o alcance da pesquisa de campo. Porém, se isso for verdadeiro, não foi possível observar, no intervalo de três anos, o final desses ciclos de investigação, com um correspondente e esperado aumento de relatos finais de pesquisas com dados de campo.

Outro possível motivo para a grande proporção de estudos teórico-conceituais é, como nos resultados de Filippini e Voss (1997), a ausência pura e simples de trabalhos com pesquisa de campo. As categorias Modelagem, Simulação e Teórico-Conceitual, isto é, sem evidências de pesquisas de campo, somam mais de $60 \%$ dos artigos publicados no período analisado. Isso não desmerece nem invalida esses trabalhos, porém, pode lançar dúvidas quanto à aplicabilidade dos modelos e resultados encontrados, pela possibilidade da falta de aderência desses resultados à realidade. Por outro lado, o simples fato da pesquisa apresentar dados de campo não garante que a investigação foi bem conduzida. Filippini e Voss afirmam que, a menos que a pesquisa empírica tenha sido realizada com metodologias bem planejadas, os resultados serão difíceis de generalizar ou reproduzir.
Muitos dos trabalhos examinados carecem de estruturação que permita a identificação do método e/ou tipo de pesquisa utilizado. O reflexo dessa ausência não se limita apenas à falta de formalidade da estrutura dos trabalhos. Em muitos casos, prejudica não somente a replicação ou generalização dos resultados, como temem Filippini e Voss (1997), mas a própria consistência dos resultados encontrados, pois a lógica de raciocínio utilizado não é devidamente explicitada.

O uso incorreto do termo "Estudo de Caso" foi, sem dúvida, a observação mais freqüente no material analisado. Diversos autores verificados declaram erroneamente em seus títulos e textos “... o caso da empresa $X X$ ”, utilizando a terminologia como figura de linguagem e não como indicação ou explicitação do tipo de pesquisa utilizado. Muitas vezes, fato do objeto de estudo referir-se à uma única empresa faz com que o rótulo "Estudo de Caso" seja utilizado, mesmo que o levantamento de dados e a análise da organização não atinjam a profundidade exigida por esse tipo de pesquisa. Esse mesmo desvio foi encontrado em estudos onde se fez uso do rótulo para definir a coleta de dados em uma única organização, para a aplicação, comprovação ou validação de métodos ou modelos diversos. Outra situação freqüente foi o uso do termo para caracterizar um setor, por exemplo: "O caso do setor hoteleiro" ou "O caso das empresas de telecomunicações", a despeito do tipo de pesquisa efetivamente aplicado não ser o Estudo de caso. Somente nesta última situação, a declaração do tipo não foi respeitada, mas procurou-se analisar o verdadeiro tipo empregado. 
PRODUÇÃO =

5. Comparação entre os resultados brasilei ros e os norte-americanos

O Decision Science Institute, de cujas conferências anuais foram extraídos os dados publicados por Filippini (1997), é um instituto multidisciplinar, criado em 1968, cujo foco principal reside na aplicação de métodos quantitativos a problemas funcionais e comportamentais em gestão de negócios (DSI, 1999). A abrangência e a diversidade dos assuntos tratados nas conferências do DSI são menores do que as do ENEGEP. Assuntos como gestão da tecnologia, gestão ambiental, construção civil, agro-indústria, projeto do produto, organização do trabalho e ergonomia, que têm significativa quantidade de artigos no ENEGEP, não são contemplados nos eventos do DSI. Desta forma, espera-se que haja, por exemplo, menor proporção de artigos de Modelagem e Simulação e maior de Estudos de Campo no ENEGEP, quando comparada ao DSI.
Colocados lado a lado, os resultados das Tabelas 2 e 3, transformam-se nos dados apresentados pela Tabela 4 .

Conforme o esperado, observou-se que Modelagem e, principalmente, Simulação apresentam número de ocorrências significativamente mais baixos, enquanto que Estudos de Campo têm número expressivamente maior. A preponderância de estudos qualitativos em áreas como estratégia, organização do trabalho e ergonomia pode explicar o maior número de Estudos de Campo.

Os estudos teórico-conceituais são muito mais numerosos nos Anais do ENEGEP do que no DSI. Algumas hipóteses sobre os motivos do número de trabalhos teórico-conceituais já foram efetuados. Cabe observar porém que, embora no período de dez anos a ocorrência desse tipo de pesquisa tenha dobrado nas conferências norteamericanas, sua proporção nos anais do evento brasileiro são ainda significativamente mais altas.

\begin{tabular}{cccccc}
\hline $\begin{array}{c}\text { Método de } \\
\text { Pesquisa }\end{array}$ & $\begin{array}{c}\text { Anais DSI } \\
\mathbf{1 9 8 6 / 8 7}\end{array}$ & $\begin{array}{c}\text { Anais DSI } \\
\text { Anais }\end{array}$ & $\begin{array}{c}\text { Anais } \\
\text { ENEGEP }\end{array}$ & $\begin{array}{c}\text { Anais } \\
\text { ENEGEP } \\
\text { ENEGEP 1998 }\end{array}$ \\
$\begin{array}{ccccc}1999 \\
\text { Modelagem }\end{array}$ & $33,96 \%$ & $27,66 \%$ & $12,69 \%$ & $16,49 \%$ & $12,45 \%$ \\
Simulação & $32,08 \%$ & $16,17 \%$ & $2,67 \%$ & $4,23 \%$ & $3,35 \%$ \\
Survey & $13,21 \%$ & $26,81 \%$ & $3,01 \%$ & $4,86 \%$ & $4,83 \%$ \\
Estudo de Caso & $7,54 \%$ & $2,13 \%$ & $16,86 \%$ & $14,16 \%$ & $13,94 \%$ \\
Estudo de Campo & $1,89 \%$ & $5,53 \%$ & $14,86 \%$ & $19,45 \%$ & $16,54 \%$ \\
Experimento & $1,89 \%$ & $0,85 \%$ & $1,50 \%$ & $2,33 \%$ & $0,74 \%$ \\
Teórico / & $9,43 \%$ & $20,85 \%$ & $48,41 \%$ & $38,48 \%$ & $48,14 \%$ \\
Conceitual & & & & & \\
\hline
\end{tabular}


No congresso brasileiro, as pesquisas do tipo "Levantamento/Survey" têm número de ocorrências significativamente mais baixo, enquanto um número muito maior de ocorrências são verificadas para Estudos de Caso. Diversos motivos podem ser levantados para essas diferenças. O primeiro deles é, sem dúvida, o uso equivocado do termo "Estudo de caso" por autores brasileiros.

A aparente flexibilidade desse tipo de pesquisa parece seduzir os pesquisadores mas sua utilização inconseqüente e isenta de aporte metodológico adequado pode comprometer e invalidar esforços e resultados.

Nas pesquisas brasileiras, a baixa utilização do survey talvez possa ser explicada pelo seu custo. A necessidade de mobilizar recursos financeiros pode ser considerado como um dos fatores preponderantes que restringem sua opção. A realização de um survey por mala direta incorre em muitos custos, que vão desde a realização da pesquisa-piloto, a postagem e o acompanhamento telefônico posterior. Além disso, também própria a operacionalização das variáveis de pesquisa, a definição de suas escalas de medida e o plano de análise de dados podem representar obstáculos ao pesquisador. Entretanto, essas dificuldades não devem restringir o seu uso, pois existem fenômenos que só podem ser corretamente abordados e conhecidos se investigados através de survey.

Os estudos de natureza experimental assim como os qualitativos do tipo pesquisa ação e pesquisa participante foram recursos metodológicos pouco utilizados pelos autores observados. É possível que a baixa ocorrência se verifique pela complexidade inerente à essas tipos ou ainda devido à sua inadequação para a solução de problemas características das áreas e sub-áreas da Engenharia de Produção. Neste caso, seria necessário a realização de outras pesquisas que comprovassem tais afirmações.

\section{Conclusões}

PRODUÇÃO

É evidente que a explosão da informação causada pela facilidade de veiculação - transmissão e recuperação - de conhecimentos através de meios eletrônicos e virtuais, aumentam as chances da disseminação de dados e informações sem qualquer filtro, caráter ou tratamento metodológico. Ao mesmo tempo, são criados mecanismos mais eficientes e rígidos de verificação e validação do desenvolvimento científico e tecnológico, que promovem em todos os campos do conhecimento humano, uma mudança estrutural na condução de pesquisas. Nas comunidades técnicas crescem as exigência pela sistematização e registro do conhecimento baseado em ciência, ou seja, produto de investigações científicas e que incluem a presença de aparato metodológico adequado, comunicação e registro de resultados.

Os resultados encontrados neste estudo apontam para um comportamento de pesquisa singular se comparados à outras áreas de pesquisa brasileiras. A princípio, há que se notar que, no Brasil, a Engenharia Produção é uma área de pesquisa relativamente nova se comparada à algumas outras ciências (médicas, exatas e sociais). Se, por um lado, isso explica e abranda a ausência de tradição no uso de métodos e técnicas de pesquisa, por outro, evidencia e aponta para a necessidade da tomada de uma posição mais contundente e ativa no ensino, disseminação e na exigência de um maior rigor metodológico. Um aspecto que reforça e intensifica essa necessidade refere-se à multidisciplinariedade característica da Engenharia de Produção (Lovejoy, 1996), que torna necessário o domínio tanto de métodos de pesquisa quantitativos como qualitativos.

Uma tendência esperada nas pesquisas é a mudança de seu foco ou intenção: da reprodução para a produção do conhecimento, onde os 
PRODUÇÃO

pesquisadores estejam mais preocupados e motivados a produzir novos conhecimentos do que compilar e reproduzir conteúdos já existentes.

Para o caso específico do Encontro Nacional de Engenharia de Produção, deve-se observar que o evento - o mais importante do gênero, no país é um saudável fórum ampliado de discussões e relatos que inclui contribuições de pesquisas de diferentes naturezas e níveis de especialização de trabalhos de graduação e trabalhos finais de disciplinas de pós-graduação, que têm o tempo disponível para a sua realização limitado, restringindo a realização de pesquisas com sustentação metodológica mais elaborada, a projetos de pesquisa e estudos de inovação com impacto internacional, envolvendo equipes de pesquisadores experimentados. Dada essa heterogeneidade, sugere-se que sejam criadas, para os próximos eventos, sessões ou categorias específicas que privilegiem os aspectos acima descritos.

Uma sugestão importante para o registro adequado do conteúdo dos trabalhos apresentados em eventos técnicos, se refere à orientação e à exigência da elaboração de resumos que indiquem além do assunto tratado, a descrição sucinta das técnicas de pesquisa utilizadas e dos resultados encontrados. Todos os aspectos e observações efetuados acima têm em comum, um forte indício da necessidade de se introduzir ou aprimorar o ensino de Metodologia de Pesquisa Científica nos cursos de graduação e pós-graduação assim como a difundir a importância de estudos e pesquisas amparados em métodos e técnicas adequados, que validem, potencializem e valorizem os resultados e os pesquisadores envolvidos.

\section{Referência Bibliográfica}

BERTO, Rosa M.V.S., NAKANO, Davi N. Métodos de pesquisa na Engenharia de Produção. In: ENCONTRO NACIONAL DE ENGENHARIA DE PRODUÇÃO, 18. Niterói, 1998. Anais. Niterói : UFF/ABEPRO, 1998. (CDROM)

CERVO, A C., BERVIAN, P. A Metodologia científica. 4.ed.. São Paulo : Makron, 1996. 203p.

DECISION SCIENCE INSTITUTE. DSI home page (http://www.dsi.gsu.edu/dsi.htm, documento html capturado em 02/06/99)

FILIPPINI, Roberto. Operations management research: some reflections on evolution, models and empirical studies in OM. International Journal of Operations and Production Management, v.17, n.7, p. 655-70, 1997. , VOSS, Chris. Editorial. International Journal of Operations and Production Management, v.17, n.7, p. 653-54, 1997.

LAKATOS, Eva M., MARCONI, Marina A. Fundamentos de metodologia científica. 3. ed.. São Paulo : Atlas, 1991. 270p.

LOVEJOY, William S. Integrated operations: a proposal for operations management teaching and research. Production and Operations Management, v.7, n.2, p. 106 - 124, Summer 1998. 
NAKANO, Davi N., FLEURY, Afonso C.

C. Métodos de pesquisa em Engenharia de Produção. In: ENCONTRO NACIONAL DE ENGENHARIA DE PRODUÇÃO, 16.

Piracicaba, 1996. Anais. Piracicaba:

UNIMEP/ABEPRO, 1996. (CD-ROM)

VOSS, C. A Operations management- from

Taylor to Toyota - and beyond? British Journal of Management, n. 6, p.17-29., Dec. 1995. 